\title{
Mit dem Vitamin-D-Wert steigt das Risiko für hellen Hautkrebs
}

- Mehr Sonne, steigende Vitamin-D-Werte, erhöhtes Risiko für nicht melanozytären Hautkrebs? In einer Kohortenstudie wurden die oft diskutierten Zusammenhänge zwischen Vitamin-D-Spiegel und Karzinomrisiko untersucht.

Zwischen 1997 und 2001 waren 3223 Teilnehmer der Studie in Einrichtungen der amerikanischen Gesundheitsvorsorge (HMO) auf Osteoporose oder eine niedrige Knochendichte hin untersucht worden. Die überwiegend weiblichen Patienten $(89,3 \%)$ waren im Mittel 65,9 Jahre alt und wurden im Schnitt 9,8 Jahre beobachtet.

Bei 2257 Patienten ergab die Laboruntersuchung einen unzureichenden Vitamin-D-Wert ( $<30 \mathrm{ng} / \mathrm{ml})$. Nur bei 966 Patienten wurden ausreichende 25-OHD-Werte ( $\geq 30 \mathrm{ng} / \mathrm{ml}$ ) gemessen. Bei 240 Patienten wurde innerhalb der Studienzeit ein nicht melanozytärer Hautkrebs (NMSC) diagnostiziert. Dabei handelte es sich in 49 Fäl-

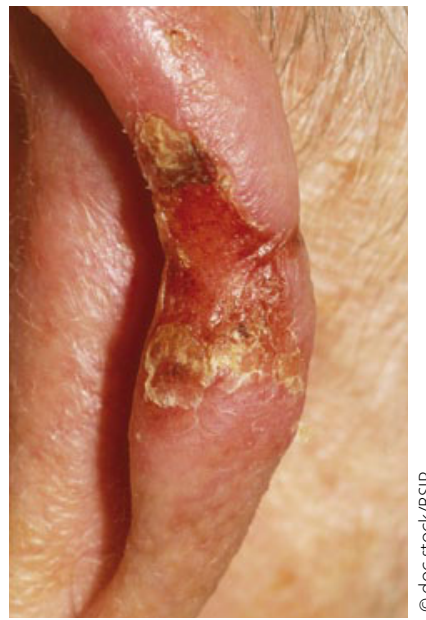

Das Ohr ist eine Prädilektionsstelle für hellen Hautkrebs. len um ein spinozelluläres Karzinom (SCC), bei 163 Patienten um ein Basalzellkarzinom (BCC) und bei 28 wurden beide Karzinomtypen nachgewiesen.

\section{Risiko beginnt schon im Normbereich}

Wurde die Höhe des VitaminD-Spiegels mit der Anzahl an Krebserkrankungen korreliert, ergab sich folgendes Bild: Bereits ab einem Wert von $15 \mathrm{ng} / \mathrm{ml}$ stieg das Risiko. Je höher der 25-OHD-Spiegel lag, desto höher war das Risiko für einen nicht melanozytären Hautkrebs (25-OHD < 19 ng/ml: OR 1; 25-OHD 19-24 ng/ml: OR 1,3; 25-OHD 25-30 ng/ $\mathrm{ml}:$ OR 1,4; $25-\mathrm{OHD} \geq 31 \mathrm{ng} / \mathrm{ml}$ : OR 1,6 bzw. adjustierte OR 1,8; $95 \%$ $\mathrm{KI} 1,1-2,9)$.

Wurden die Krebsarten im Einzelnen betrachtet, ergaben sich zwar keine signifikanten Unterschiede, es zeichneten sich aber dennoch erhöhte Risiken für Patienten mit höheren 25-OHD-Spiegeln ab: SCC adjustierte OR 1,8; 95\% KI 1,1-2,9 und BCC adj. OR 1,7; 95\% KI $1,0-2,9$. Auch bei der Untersuchung der weniger UV-exponierten Körperstellen zeigte sich ein nicht signifikanter Anstieg des NMSCRisikos mit dem 25-OHD-Wert $(2,2 ; 95 \% \mathrm{KI} 0,7-7,0)$.

Um den Einfluss wichtiger Störfaktoren auszuschließen, halten die Studienautoren eine repräsentative Untersuchung für notwendig, bei der sowohl Informationen über das Ausmaß der UV-Exposition und weitere Risikofaktoren als auch über Essgewohnheiten und eine Vitamin-D-Supplementation mit berücksichtigt werden. ST .

Eide M. J. et al. Vitamin D and Nonmelanoma Skin Cancer in a Health Maintenance Organsization Cohort. Archives of Dermatology 2011; online first Aug 15, 2011

\section{ACHTUNG:}

\section{Hier muss der Dummy durch eine Anzeige ersetzt werden !!}

